\title{
DETERMINING A SEMILINEAR PARABOLIC PDE FROM FINAL DATA *
}

\author{
Luis A. Fernández \\ Dep. de Matemáticas, Estadística y Computación \\ Universidad de Cantabria. 39071 - Santander (SPAIN) \\ lafernandez@unican.es
}

Cecilia Pola

Dep. de Matemáticas, Estadística y Computación

Universidad de Cantabria. 39071 - Santander (SPAIN)

polac@unican.es

Abstract Given the following boundary value problem

$$
\begin{cases}y_{t}-\Delta y+a(x) b(y)=u \chi_{\omega_{i n}} & \text { in } \Omega \times(0, T) \\ y=0 & \text { on } \partial \Omega \times(0, T), \\ y(0)=y_{0} & \text { in } \Omega,\end{cases}
$$

we are concerned with the following inverse problem: assuming that for each source term $u(x, t) \in L^{2}\left(\omega_{i n} \times(0, T)\right)$ the solution at the final time $y(x, T)$ is known over $\omega_{\text {out }}$, we want to determine the nonlinear term $a(x) b(y)$ over $\Omega \times \mathbb{R}$. We suppose that $\omega_{i n}$ and $\omega_{\text {out }}$ are (probably small) fixed open subsets of $\Omega$ with $\omega_{i n} \cap \omega_{\text {out }} \neq \emptyset$ and $\chi_{\omega_{\text {in }}}$ denotes the characteristic function of $\omega_{i n}$.

Keywords: inverse problem, semilinear parabolic PDE, parameter estimation.

\section{Introduction}

Let us consider the following boundary value problem, associated with a semilinear parabolic equation

$$
\begin{cases}y_{t}(x, t)-\Delta y(x, t)+a(x) b(y(x, t))=u(x, t) \chi_{\omega_{i n}}(x) & \text { in } Q \\ y(x, t)=0 & \text { on } \Sigma \\ y(x, 0)=y_{0}(x) & \text { in } \Omega\end{cases}
$$

${ }^{*}$ This work was partially supported by DGES (Spain). Proyect PB $98-0193$.

The original version of this chapter was revised: The copyright line was incorrect. This has been corrected. The Erratum to this chapter is available at DOI: 10.1007/978-0-387-35690-7_44 
where $\Omega$ is a bounded open subset of $\mathbb{R}^{n}$ with a boundary $\partial \Omega$ of class $C^{2}, Q=\Omega \times(0, T), \Sigma=\partial \Omega \times(0, T)$ and $T>0$.

Given the functions $a, b, u$ and $y_{0}$, the classical direct problem consists of determining $y(x, t)$ over $Q$. Here we are concerned with the following inverse problem: assuming that the initial datum $y_{0}$ is known and that for each source term $u \in L^{2}\left(\omega_{i n} \times(0, T)\right)$ the solution at the final time $y(x, T)$ is known over $\omega_{\text {out }} \subset \Omega$, we want to determine the nonlinear term $a(x) b(y)$.

Our work is somehow related to the recovery of a nonlinear term $f(x, t, y)$ through the knowledge of the Dirichlet-to-Neumann map associated to the semilinear PDE, see [4, Chapter 9] and the bibliography therein.

On the other hand, in [2], we have studied the case where the source terms $u$ are acting on the whole domain $Q$ (i.e. $\omega_{i n}=\Omega$ ). In fact, in that case, we have proved the simultaneous identification of the nonlinearities $A, \vec{b}$ and $c$ appearing in the quasilinear parabolic equation

$$
y_{t}-\operatorname{div}(A(y) \nabla y+\vec{b}(y))+c(y)=u(x, t) \text { in } Q,
$$

that are unique in an appropriate class.

The main objective of this work is to deal with the case $\omega_{i n} \neq \Omega$. To this end, we restrict ourselves to the semilinear PDE given in (1), although the linear part of the operator can be taken in a more general way. In the first part of the work, we prove the uniqueness of $a(x) b(y)$ assuming that $a$ is analytic on $\bar{\Omega}$ without changing the sign and $b \in$ $C^{1}(\mathbb{R})$ with $b^{\prime} \in L^{\infty}(\mathbb{R})$. This property can be deduced by using the approximate controllability for this type of equations, when the function $u$ is viewed as a control.

In the second part, we present a finite-dimensional optimization problem that allows the practical reconstruction of $a(x) b(y)$. In order to illustrate the applicability of the method, some numerical results are shown in the one-dimensional space case (i.e. $n=1$ ). The influence of noise in the data will be also taken into account.

\section{Uniqueness of the nonlinear term}

Let us recall the space

$$
W(0, T)=\left\{y \in L^{2}\left(0, T ; H_{0}^{1}(\Omega)\right): y_{t} \in L^{2}\left(0, T ; H^{-1}(\Omega)\right)\right\},
$$

where $H_{0}^{1}(\Omega)$ denotes the classical Sobolev space and $H^{-1}(\Omega)$ its dual.

Let us introduce the hypotheses that will be assumed on the nonlinear term of the semilinear parabolic operator:

H1) $a$ is a real analytic function on $\bar{\Omega}$ and $a(x) \geq 0 \quad \forall x \in \bar{\Omega}$.

H2) $b \in C^{1}(\mathbb{R})$ with $b^{\prime} \in L^{\infty}(\mathbb{R})$. 
The sign condition in H1) is just made to fix ideas: the important point here is that $a$ does not change the sign in $\Omega$ (see the end of the proof of Theorem 2).

For the sake of brevity, we designate $\mathcal{C}=\{(a, b)$ verifying $H 1)-H 2)\}$.

Given $(a, b) \in \mathcal{C}, u \in L^{2}\left(\omega_{i n} \times(0, T)\right)$ and $y_{0} \in L^{2}(\Omega)$, it is well known that the problem (1) has a unique solution $y$ in $W(0, T)$, see [5, Theorem 6.7, pp. 466]. Taking into account that the initial datum $y_{0}$ will remain fixed through the rest of the paper, we will designate by $y_{u, a, b}$ the unique solution of problem (1) in $W(0, T)$.

Let us now recall an approximate controllability result for parabolic equations:

Theorem 1 Let us fix $y_{0} \in L^{2}(\Omega),(a, b) \in \mathcal{C}$ and $\omega_{\text {in }}$ a nonempty open subset of $\Omega$. Then, the set $R(T)=\left\{y_{u, a, b}(x, T): u \in L^{2}\left(\omega_{i n} \times(0, T)\right)\right\}$ is dense in $L^{2}(\Omega)$.

This is a particular case of a more general result (see for instance [1] and [3]). At this level, the assumption $\mathrm{H} 1$ ) on $a$ can be weakened to $a \in L^{\infty}(\Omega)$.

With the help of this controllability result, we can establish the following uniqueness theorem:

Theorem 2 (Uniqueness) Let us fix $y_{0} \in L^{2}(\Omega)$ and $\omega_{\text {in }}, \omega_{\text {out }}$ two open subsets of $\Omega$ such that $\omega_{\text {in }} \cap \omega_{\text {out }} \neq \emptyset$. Suppose that there exist two pairs $(a, b),(\tilde{a}, \tilde{b}) \in \mathcal{C}$ such that $y_{u, a, b}(T)=y_{u, \tilde{a}, \tilde{b}}(T)$ in $\omega_{\text {out }}$ for each $u \in L^{2}\left(\omega_{\text {in }} \times(0, T)\right)$. Then, $a(x) b(s)=\tilde{a}(x) \tilde{b}(s)$ for all $(x, s) \in \Omega \times \mathbb{R}$.

Proof. Given $u, v \in L^{2}\left(\omega_{i n} \times(0, T)\right)$ and $\lambda \in(0,1)$, let us consider $y_{u+\lambda v, a, b}$ and $y_{u, a, b}$. Moreover we define $z_{\lambda, a, b}=\left(y_{u+\lambda v, a, b}-y_{u, a, b}\right) / \lambda$. Analogously, from $y_{u+\lambda v, \tilde{a}, \tilde{b}}$ and $y_{u, \tilde{a}, \tilde{b}}$ we introduce $z_{\lambda, \tilde{a}, \tilde{b}}=\left(y_{u+\lambda v, \tilde{a}, \tilde{b}}-\right.$ $y_{u, \tilde{a}, \tilde{b}} / \lambda$. By hypothesis we know that $y_{u, a, b}(T)=y_{u, \tilde{a}, \tilde{b}}(T)$ in $\omega_{\text {out }}$ and $y_{u+\lambda v, a, b}(T)=y_{u+\lambda v, \tilde{a}, \tilde{b}}(T)$ in $\omega_{o u t}$ for each $\lambda$. Consequently, $z_{\lambda, a, b}(T)=$ $z_{\lambda, \tilde{a}, \tilde{b}}(T)$ in $\omega_{\text {out }}$. By using the Mean Value Theorem, it can be proved that $z_{\lambda, a, b} \rightarrow z_{v, a, b}$ in $W(0, T)$ as $\lambda \rightarrow 0$, where $z_{v, a, b}$ is the unique solution in $W(0, T)$ of the problem

$$
\begin{cases}z_{t}(x, t)-\Delta z(x, t)+a(x) b^{\prime}\left(y_{u, a, b}\right) z(x, t)=v(x, t) \chi_{\omega_{i n}}(x) & \text { in } Q, \\ z(x, t)=0 & \text { on } \Sigma, \\ z(x, 0)=0 & \text { in } \Omega .\end{cases}
$$

Thanks to H2), let us point out that $a(x) b^{\prime}\left(y_{u, a, b}(x, t)\right) \in L^{\infty}(Q)$. Hence, it is clear that previous problem is well posed in $W(0, T)$. 
The same argumentation leads us to show that $z_{\lambda, \tilde{a}, \tilde{b}} \rightarrow z_{v, \tilde{a}, \tilde{b}}$ in $W(0, T)$ as $\lambda \rightarrow 0$, where $z_{v, \tilde{a}, \tilde{b}}$ is the unique solution in $W(0, T)$ of the problem

$$
\begin{cases}z_{t}(x, t)-\Delta z(x, t)+\tilde{a}(x) \tilde{b}^{\prime}\left(y_{u, \tilde{a}, \tilde{b}}\right) z(x, t)=v(x, t) \chi_{\omega_{i n}}(x) & \text { in } Q, \\ z(x, t)=0 & \text { on } \Sigma, \\ z(x, 0)=0 & \text { in } \Omega .\end{cases}
$$

Furthermore, $z_{v, a, b}(T)=z_{v, \tilde{a}, \tilde{b}}(T)$ in $\omega_{\text {out }}$. Now, we introduce $w=$ $z_{v, a, b}-z_{v, \tilde{a}, \tilde{b}}$ that satisfies $w(T)=0$ in $\omega_{\text {out }}$ and it can be viewed as the unique solution in $W(0, T)$ of the linear problem

$$
\begin{cases}w_{t}-\triangle w+a b^{\prime}\left(y_{u, a, b}\right) w=\left(\tilde{a} \tilde{b}^{\prime}\left(y_{u, \tilde{a}, \tilde{b}}\right)-a b^{\prime}\left(y_{u, a, b}\right)\right) z_{v, \tilde{a}, \tilde{b}} & \text { in } Q \\ w(x, t)=0 & \text { on } \Sigma \\ w(x, 0)=0 & \text { in } \Omega\end{cases}
$$

For each $\phi \in L^{2}\left(\omega_{\text {out }}\right)$, we consider the following adjoint problem:

$$
\begin{cases}-p_{t}(x, t)-\Delta p(x, t)+a(x) b^{\prime}\left(y_{u, a, b}(x, t)\right) p(x, t)=0 & \text { in } Q \\ p(x, t)=0 & \text { on } \Sigma, \\ p(x, T)=\phi(x) \chi_{\omega_{\text {out }}}(x) & \text { in } \Omega,\end{cases}
$$

whose unique solution $p_{\phi}$ also belongs to $W(0, T)$.

Multiplying the equation (2) by $p_{\phi}$, integrating by parts and taking into account that $w(T) p(T)=0$ in $\Omega$, we obtain

$$
\int_{Q}\left(\tilde{a}(x) \tilde{b}^{\prime}\left(y_{u, \tilde{a}, \tilde{b}}(x, t)\right)-a(x) b^{\prime}\left(y_{u, a, b}(x, t)\right)\right) z_{v, \tilde{a}, \tilde{b}}(x, t) p_{\phi}(x, t) d x d t=0,
$$

for all $v \in L^{2}\left(\omega_{\text {in }} \times(0, T)\right)$ and all $\phi \in L^{2}\left(\omega_{\text {out }}\right)$.

Finally, let us introduce a new boundary problem associated with the previous ones, given by

$$
\begin{cases}-q_{t}-\triangle q+\tilde{a} \tilde{b}^{\prime}\left(y_{u, \tilde{a}, \tilde{b}}\right) q=\left(\tilde{a} \tilde{b}^{\prime}\left(y_{u, \tilde{a}, \tilde{b}}\right)-a b^{\prime}\left(y_{u, a, b}\right)\right) p_{\phi} & \text { in } Q \\ q(x, t)=0 & \text { on } \Sigma \\ q(x, T)=0 & \text { in } \Omega\end{cases}
$$

Once more, multiplying the PDE in (5) by $z_{v, \tilde{a}, \tilde{b}}$, integrating by parts, taking into account (4) and the PDE satisfied by $z_{v, \tilde{a}, \tilde{b}}$, we derive

$$
\int_{Q} v(x, t) \chi_{\omega_{i n}}(x) q(x, t) d x d t=0, \text { for all } v \in L^{2}\left(\omega_{i n} \times(0, T)\right) .
$$


Therefore, $q(x, t)=0$ in $\omega_{i n} \times(0, T)$ and using the PDE in (5) we get

$$
\left(\tilde{a}(x) \tilde{b}^{\prime}\left(y_{u, \tilde{a}, \tilde{b}}(x, t)\right)-a(x) b^{\prime}\left(y_{u, a, b}(x, t)\right)\right) p_{\phi}(x, t)=0,
$$

in $\omega_{\text {in }} \times(0, T)$, for all $\phi \in L^{2}\left(\omega_{\text {out }}\right)$.

In particular, taking $t=T$ in (7), we have

$$
\left(\tilde{a}(x) \tilde{b}^{\prime}\left(y_{u, \tilde{a}, \tilde{b}}(x, T)\right)-a(x) b^{\prime}\left(y_{u, a, b}(x, T)\right)\right) \phi(x) \chi_{\omega_{\text {out }}}(x)=0 \text { in } \omega_{\text {in }}
$$

for all $\phi \in L^{2}\left(\omega_{\text {out }}\right)$. Therefore,

$$
\tilde{a}(x) \tilde{b}^{\prime}\left(y_{u, \tilde{a}, \tilde{b}}(x, T)\right)=a(x) b^{\prime}\left(y_{u, a, b}(x, T)\right) \quad \text { in } \omega_{\text {in }} \cap \omega_{\text {out }} .
$$

Taking into account that $y_{u, a, b}(T)=y_{u, \tilde{a}, \tilde{b}}(T)$ in $\omega_{\text {out }}$ for each $u$ in $L^{2}\left(\omega_{i n} \times(0, T)\right)$ and that Theorem 1 implies the density of

$$
\left\{y_{u, a, b}(T)_{\mid \omega_{i n} \cap \omega_{\text {out }}}: u \in L^{2}\left(\omega_{\text {in }} \times(0, T)\right)\right\}
$$

in $L^{2}\left(\omega_{\text {in }} \cap \omega_{\text {out }}\right)$, for each $s \in \mathbb{R}$ we can select a sequence $\left\{u_{m}\right\} \subset$ $L^{2}\left(\omega_{i n} \times(0, T)\right)$ such that as $m$ tends to $+\infty$

$$
y_{u_{m}, a, b}(T) \longrightarrow s \text { in } L^{2}\left(\omega_{\text {in }} \cap \omega_{\text {out }}\right) .
$$

Using the identity (8) for each $u_{m}$ and passing to the limit in $m$, we arrive to $\tilde{a}(x) \tilde{b}^{\prime}(s)=a(x) b^{\prime}(s)$ for all $(x, s) \in\left(\omega_{\text {in }} \cap \omega_{\text {out }}\right) \times \mathbb{R}$. When $\tilde{a}(x) \neq 0$ and $b^{\prime}(s) \neq 0$, we derive the existence of a real constant $C_{1}$ such that

$$
\frac{a(x)}{\tilde{a}(x)}=\frac{\tilde{b}^{\prime}(s)}{b^{\prime}(s)}=C_{1} \quad \forall x \in \omega_{\text {in }} \cap \omega_{\text {out }}, \forall s \in \mathbb{R} .
$$

Due to the unique analytic continuation property, $a(x)=C_{1} \tilde{a}(x)$ for all $x \in \Omega$. Moreover, there exists a real constant $C_{2} \in \mathbb{R}$ such that $\tilde{b}(s)=C_{1} b(s)+C_{2}$ for all $s \in \mathbb{R}$. Let us point out that $\tilde{a}(x) \tilde{b}(s)=$ $a(x) b(s)+C_{2} \tilde{a}(x)$ for all $(x, s) \in \Omega \times(0, T)$.

To show that $C_{2}=0$, we fix $u \in L^{2}\left(\omega_{i n} \times(0, T)\right)$ and introduce $\zeta=y_{u, a, b}-y_{u, \tilde{a}, \tilde{b}}$. Arguing as at the beginning of the proof, we derive that $\zeta$ is the unique solution of the following linear problem:

$$
\begin{cases}\zeta_{t}(x, t)-\Delta \zeta(x, t)+a(x) c(x, t) \zeta(x, t)=C_{2} \tilde{a}(x) & \text { in } Q, \\ \zeta(x, t)=0 & \text { on } \Sigma, \\ \zeta(x, 0)=0 & \text { in } \Omega,\end{cases}
$$

where $c(x, t)=\int_{0}^{1} b^{\prime}\left(s y_{u, a, b}(x, t)+(1-s) y_{u, \tilde{a}, \tilde{b}}(x, t)\right) d s$. Moreover, by hypothesis we know that $\zeta(x, T)=0$ in $\omega_{\text {out }}$. Once more, given $\phi \in$ $L^{2}\left(\omega_{\text {out }}\right)$, we consider $\hat{p}$ the unique solution of the adjoint problem 


$$
\begin{cases}-\hat{p}_{t}(x, t)-\Delta \hat{p}(x, t)+a(x) c(x, t) \hat{p}(x, t)=0 & \text { in } Q, \\ \hat{p}(x, t)=0 & \text { on } \Sigma, \\ \hat{p}(x, T)=\phi(x) \chi_{\omega_{\text {out }}}(x) & \text { in } \Omega .\end{cases}
$$

Multiplying by $\hat{p}$ the PDE satisfied by $\zeta$, integrating by parts and using that $\hat{p}(T) \zeta(T)=0$ in $\Omega$, we deduce

$$
C_{2} \int_{Q} \tilde{a}(x) \hat{p}(x, t) d x d t=0 .
$$

Choosing $\phi(x) \geq 0$ for all $x \in \omega_{\text {out }}, \phi \not \equiv 0$, the Maximum Principle implies $\hat{p}(x, t) \geq 0$ in $Q, \quad \hat{p} \not \equiv 0$. Noticing that $\tilde{a}(x) \geq 0$ in $\Omega$, the equality (11) implies $C_{2}=0$.

\section{Remarks.}

i) It follows from the proof that the functions $a(x)$ and $b(y)$ can be determined separately in a unique way, up to a multiplicative constant, because $a(x) b(y)=\frac{a(x)}{C_{1}} C_{1} b(y)$ for all $C_{1}>0$.

ii) Let us point out that the conclusion of Theorem 2 remains valid if it is supposed that the relation $y_{u, a, b}(T)=y_{u, \tilde{a}, \tilde{b}}(T)$ in $\omega_{\text {out }}$ only holds when $u \in L^{2}\left(\omega_{i n} \times[T-\delta, T]\right)$, where $\delta \in(0, T)$ is fixed. This is a consequence of the fact that the controllability result also holds in this case, i.e. under the conditions of Theorem 1, the set $R(T)=\left\{y_{u, a, b}(x, T): u \in L^{2}\left(\omega_{i n} \times[T-\delta, T]\right)\right\}$ is dense in $L^{2}(\Omega)$, (see [3, Remark 3.1 (c)]). Here, $y_{u, a, b}$ denotes the unique solution of problem (1), when the right hand term of the PDE is given by $u(x, t) \chi_{\omega_{i n} \times[T-\delta, T]}(x, t)$.

iii) Exactly the same technique can be used to prove the uniqueness of the term $a(x) y+b(y)$ for the Dirichlet boundary problem associated to the PDE $y_{t}-\triangle y+a(x) y+b(y)=u \chi_{\omega_{i n}}$, under the assumptions that $a$ is analytic on $\bar{\Omega}$ and H2) (no sign condition has to be imposed on $a$ ).

\section{The identification process. Numerical experiments.}

In this section, problem (1) is considered in the one-dimensional space case (i.e. $n=1$ ). We are concerned with getting a numerical approximation $\check{a}(x) \breve{b}(y)$ of the nonlinear term $a(x) b(y)$ from a finite number of observations $\eta_{i j}$. Let us consider (1) for a finite set of source terms $\left\{u_{j}\right\}_{j=1}^{n_{u}}$. For each $u_{j}$, we have measurements $\eta_{i j}$ of $y_{u_{j}, a, b}\left(x_{i}, T\right)$ at some points $x_{i} \in \omega_{\text {out }}$. 
For our experiments we have chosen $y_{0}(x)=0.5 x(4-x), T=1$ and $\Omega=(0,4)$ with the grid $\left\{x_{i}=i / 10\right\}_{i=0}^{40}$. The data $\eta_{i j}$ correspond with the nodes $x_{i}$ in $\omega_{\text {out }}=(0.4-1 . e-12,2+1 . e-12)$ and they were obtained solving (1) with six of the following source terms:

$$
u_{j}(x, t)= \begin{cases}-50\left(i_{j}-2 k_{j}\right) \sin \left(i_{j} \pi t\right) \sin \left(2 k_{j} \pi x\right) & \text { if } x \in(0,0.5), \\ 0 & \text { if } x \in[0.5,4),\end{cases}
$$

for $i_{j}, k_{j} \in\{1,2 \ldots, 15\}$. Note that $\omega_{i n}=(0,0.5)$ and only the node $x_{i}=0.4$ belongs to $\omega_{\text {in }} \cap \omega_{\text {out }}$.

The first step of the identification process is related with the choice of the interval (for the variable $y$ ) where we are going to recover the nonlinearity $a(x) b(y)$. Our working interval is an enlargement of the interval defined by the data, $I=\left[\eta_{\min }=\min \eta_{i j}, \eta_{\max }=\max \eta_{i j}\right]$, by taking a safety barrier, $M>0$, in both extremes: $I_{w}=\left[\eta_{\min }-M, \eta_{\max }+M\right]$. In our calculations we took an equidistant grid $\left\{y_{i}\right\}_{y=0}^{n_{y}}$ in $I_{w}$ with a mesh size $h=\left(\eta_{\max }-\eta_{\min }\right) / 10$.

The reconstruction of $a(x) b(y)$ consists in determining two vectors $\hat{a}=\left(\hat{a}_{0}, \ldots, \hat{a}_{n_{x}}\right) \in \mathbb{R}^{n_{x}+1}$ and $\hat{b}=\left(\hat{b}_{0}, \ldots, \hat{b}_{n_{y}}\right) \in \mathbb{R}^{n_{y}+1}$, where $\hat{a}_{i}$ and $\hat{b}_{i}$ are the coefficients of the approximations

$$
\check{a}(x)=\sum_{i=0}^{n_{x}} \hat{a}_{i} B_{i}(x) \quad \text { and } \quad \check{b}(y)=\sum_{i=0}^{n_{y}} \hat{b}_{i} \hat{B}_{i}(y),
$$

being $B_{i}$ and $\hat{B}_{i}$ piecewise linear B-splines verifying $B_{i}\left(x_{2 j}\right)=\delta_{i j}$ for $i, j=0, \ldots, n_{x} \quad$ and $\quad \hat{B}_{i}\left(y_{j}\right)=\delta_{i j}$ for $i, j=0, \ldots, n_{y}$.

Let us point out that we use double mesh size for the function $\check{a}$ than for the discretization of $\Omega$. For inverse problems, some experiments indicate that it is better to take a bigger mesh size for the spatially varying parameters than for the solution $y$.

Now we are ready to state the finite-dimensional problem for recovering $a(x) b(y)$. Following an output least squares method with regularization, we consider the minimization problem

$$
\min _{(\hat{a}, \hat{b}) \in U_{a d}} J(\hat{a}, \hat{b}),
$$

where

$$
\begin{gathered}
J(\hat{a}, \hat{b})=\frac{1}{2} \sum_{j=1}^{n_{u}} \int_{\omega_{\text {out }}}\left(y_{u_{j}, \check{a}, \check{b}}(x, T)-\eta_{j}(x)\right)^{2} d x+ \\
+\gamma\left(\int_{\Omega \times I_{w}}\|\nabla(\check{a}(x) \check{b}(y))\|_{2}^{2} d x d y+\epsilon\left(\int_{\Omega}(\check{a}(x))^{2} d x+\int_{I_{w}}(\check{b}(y))^{2} d y\right)\right),
\end{gathered}
$$


being $\eta_{j}(x)$ a continuous observation which interpolates the data $\eta_{i j}$, $i=4, \ldots, 20, \gamma>0, \epsilon \geq 0$ and $U_{a d}$ the set of feasible vectors given by

$$
U_{a d}=\left\{(\hat{a}, \hat{b}) \in \mathbb{R}^{n_{x}+1} \times \mathbb{R}^{n_{y}+1}: \hat{a}_{i} \geq 0, \quad i=0, \ldots, n_{x}\right\} .
$$

Hence, the objective function is the sum of a least squares term and a regularization term. If $\epsilon>0$, the last term is useful for proving the coercivity of the functional. On the other hand, if $\epsilon=0$, we also can obtain the coercivity of $J$ by imposing some constraints, for instance: $\check{a}\left(x_{0}\right)^{2}+\check{a}\left(x_{n_{x}}\right)^{2} \leq c_{1}, \breve{b}\left(y_{0}\right)^{2}+\check{b}\left(y_{n_{y}}\right)^{2} \leq c_{2},\|\hat{a}\|^{2} \geq c_{3}>0$ and $\|\hat{b}\|^{2} \geq$ $c_{3}>0$; but this kind of formulation needs some a priori knowledge about the functions to recover. In the simpler case $a(x) \equiv 1$, taking $\breve{b}\left(y_{0}\right)=0$, $\check{b}\left(y_{n_{y}}\right)=0, J$ is coercive on $U_{a d}=\mathbb{R}^{n_{y}-1}$ (see [2]).

The following result states the existence of minimizers.

Theorem 3 There exists at least one solution to the problem (14).

Proof. Because of $J$ is continuous (see Appendix of [2]), coercive and the feasible set $U_{a d}$ is non-empty and closed, the result follows.

Problem (14) was solved by using the subroutine E04UCF from NAG Library, that implements a sequential quadratic programming (SQP) algorithm. Each evaluation of the objective function was computed by taking (for each $j$ ) a linear spline which interpolates the values $\eta_{i j}-y_{u_{j}, \check{a}, \breve{b}}\left(x_{i}, T\right)$, where $y_{u_{j}, \check{a}, \breve{b}}\left(x_{i}, T\right)$ were obtained by solving (1) with a linearized Crank-Nicholson-Galerkin method; to this end, we took a semidiscrete approach with 39 piecewise linear finite elements for the discretization of the spatial domain and the nodes $\{i T / 10\}_{i=0}^{10}$ for the discretization of the time variable.

About the stopping test, let us mention that the optimization algorithm terminates successfully if the following conditions are satisfied,

$$
\begin{array}{r}
\frac{\left\|\left(\hat{a}^{k}-\hat{a}^{k-1}, \hat{b}^{k}-\hat{b}^{k-1}\right)\right\|}{1+\left\|\left(\hat{a}^{k}, \hat{b}^{k}\right)\right\|}<\sqrt{\varepsilon}, \\
\frac{\left\|\left(\nabla J\left(\hat{a}^{k}, \hat{b}^{k}\right)\right)_{F R}\right\|}{1+\max \left\{1+\left|J\left(\hat{a}^{k}, \hat{b}^{k}\right)\right|,\left\|\left(\nabla J\left(\hat{a}^{k}, \hat{b}^{k}\right)\right)_{F R}\right\|\right\}} \leq \sqrt{\varepsilon} \\
\left(\nabla J\left(\hat{a}^{k}, \hat{b}^{k}\right)\right)_{L} \geq 0,
\end{array}
$$

where $\left(\nabla J\left(\hat{a}^{k}, \hat{b}^{k}\right)\right)_{F R}$ is the vector with the components of $\nabla J\left(\hat{a}^{k}, \hat{b}^{k}\right)$ corresponding to the free variables (i.e. not fixed at the bounds) and $\left(\nabla J\left(\hat{a}^{k}, \hat{b}^{k}\right)\right)_{L}$ is formed by the rest of the components of $\nabla J\left(\hat{a}^{k}, \hat{b}^{k}\right)$.

Concerning the bound conditions described in H1) and H2), let us point out that the lower bounds in (16) ensure $\check{a}^{k}(x) \geq 0$ for all $k$ 
and all $x \in \Omega$. On the other hand, from $J\left(\hat{a}^{k}, \hat{b}^{k}\right) \leq J\left(\hat{a}^{0}, \hat{b}^{0}\right)$, when $\int_{\Omega}\left(\check{a}^{k}(x)\right)^{2} d x \geq c_{1}>0$, it follows that

$$
\left(\left(\check{b}^{k}\right)^{\prime}(y)\right)^{2}=\left(\sum_{i=0}^{n_{y}} \hat{b}_{i} \hat{B}_{i}^{\prime}(y)\right)^{2} \leq \frac{J\left(\hat{a}^{0}, \hat{b}^{0}\right)}{\gamma h c_{1}},
$$

for all $y \notin\left\{y_{0}, \ldots, y_{n_{y}}\right\}$ and for all $k$.

Our numerical experiments were carried out in MATLAB 6 on a DELL PowerEdge 2500 with 1 GB of memory and running under Red Hat Linux, taking $(0.5, \ldots, 0.5)$ as the starting point.

To generate the observations $\eta_{i j}$, we solve (1) and take

$$
\eta_{i j}=\left(1+\delta_{i j}\right) y_{u_{j}, a, b}\left(x_{i}, T\right)
$$

where $\delta_{i j}$ are uniformly distributed random numbers in $[-\delta, \delta]$, with $\delta$ denoting the noise level.

We have considered two examples.

Example 1 Knowing $a(x) \equiv 1$ in $\Omega$, we try to recover $b(y)=3 \exp \left(-y^{2}\right)$ from the data obtained with six source terms $u_{j}$ given by (12) with $\left(i_{j}, k_{j}\right)$ taking the values $(1,1),(1,7),(2,2),(2,3),(2,4)$ and $(2,6)$. In Table 1 and Figure 1 we present the computed results obtained taking $\epsilon=0, b\left(y_{0}\right)=0$ and $b\left(y_{n_{y}}\right)=0$ in the formulation of problem (14), $\varepsilon=1 . e-9$ for the stopping test. Table 1 summarizes numerical results corresponding to $\delta=0$ (without explicit data perturbation) and $\delta=0.1$ (noisy data). For each value of $\delta$, the nodes in $I_{w}$ are listed in the first column (4 nodes in the safety barrier and 11 nodes on the interval $I$ ). For each node $y_{i}$, we report the value of the numerical solution, $\check{b}\left(y_{i}\right)$, and the value of the exact solution, $b\left(y_{i}\right)$, in the second and third column respectively, and finally the relative error $\left|\breve{b}\left(y_{i}\right)-b\left(y_{i}\right)\right| / \max \left(1,\left|b\left(y_{i}\right)\right|\right)$. At the bottom of the table we show the current values of the penalty parameter $\gamma$ and the number of iterations required by the optimization routine.

Figure 1 contains two graphics. The nonlinear term $b(y)$ obtained from data with $\delta=0$ and $\delta=0.1$ appears on the left and on the right, respectively. Each graphic shows the exact solution $b$ and the numerically identified solution $\breve{b}$. We asterisk the values $\breve{b}\left(y_{i}\right)$. Moreover, in each graphic we show the data $\eta_{i j}$ on the axis. Remark that these observations depend on the source terms $u_{j}$. Here, the main point is to choose $u_{j}$ in such a way that the corresponding observations fill as much as possible the interval where we want to recover the coefficient. Hence, it is our experience that, with a good choice, a small number of functions can be enough to recover the nonlinearity. 
Table 1. Example 1

\begin{tabular}{||c|c|c|c||c|c|c|c||}
\hline \multicolumn{4}{||c||}{ Undisturbed data $(\delta=0)$} & \multicolumn{3}{c||}{ Noisy data $(\delta=0.1)$} \\
\hline \hline$y_{i}$ & $\grave{b}\left(y_{i}\right)$ & $b\left(y_{i}\right)$ & Error & $y_{i}$ & $\grave{b}\left(y_{i}\right)$ & $b\left(y_{i}\right)$ & Error \\
\hline-1.8744 & 0.0309 & 0.0894 & $5.854 \mathrm{e}-2$ & -1.9181 & -0.0374 & 0.0757 & $1.132 \mathrm{e}-1$ \\
-1.5088 & 0.4179 & 0.3080 & $1.099 \mathrm{e}-1$ & -1.5362 & 0.2514 & 0.2832 & $3.181 \mathrm{e}-2$ \\
\hline-1.1431 & 0.7212 & 0.8121 & $9.095 \mathrm{e}-2$ & -1.1544 & 0.8193 & 0.7914 & $2.788 \mathrm{e}-2$ \\
-0.7775 & 1.6366 & 1.6390 & $1.465 \mathrm{e}-3$ & -0.7725 & 1.7270 & 1.6518 & $4.556 \mathrm{e}-2$ \\
-0.4119 & 2.5548 & 2.5319 & $9.043 \mathrm{e}-3$ & -0.3906 & 2.6902 & 2.5755 & $4.455 \mathrm{e}-2$ \\
-0.0463 & 3.0884 & 2.9936 & $3.166 \mathrm{e}-2$ & -0.0088 & 2.9227 & 2.9998 & $2.568 \mathrm{e}-2$ \\
0.3194 & 2.7510 & 2.7091 & $1.546 \mathrm{e}-2$ & 0.3731 & 2.6009 & 2.6101 & $3.526 \mathrm{e}-3$ \\
0.6850 & 1.8653 & 1.8765 & $5.932 \mathrm{e}-3$ & 0.7550 & 1.8487 & 1.6965 & $8.968 \mathrm{e}-2$ \\
1.0506 & 0.9815 & 0.9948 & $1.336 \mathrm{e}-2$ & 1.1369 & 0.8444 & 0.8238 & $2.064 \mathrm{e}-2$ \\
1.4163 & 0.3796 & 0.4037 & $2.407 \mathrm{e}-2$ & 1.5188 & 0.1584 & 0.2988 & $1.404 \mathrm{e}-1$ \\
1.7819 & 0.0994 & 0.1254 & $2.599 \mathrm{e}-2$ & 1.9006 & 0.0732 & 0.0810 & $7.731 \mathrm{e}-3$ \\
2.1475 & 0.0363 & 0.0298 & $6.480 \mathrm{e}-3$ & 2.2825 & 0.0862 & 0.0164 & $6.976 \mathrm{e}-2$ \\
2.5131 & -0.0011 & 0.0054 & $6.491 \mathrm{e}-3$ & 2.6644 & 0.1814 & 0.0025 & $1.789 \mathrm{e}-1$ \\
\hline 2.8788 & 0.0102 & 0.0008 & $9.404 \mathrm{e}-3$ & 3.0463 & 0.6878 & 0.0003 & $6.875 \mathrm{e}-1$ \\
3.2444 & -0.0106 & 0.0001 & $1.072 \mathrm{e}-2$ & 3.4281 & 0.5840 & 0.0000 & $5.840 \mathrm{e}-1$ \\
\hline \multicolumn{3}{|c|}{$\gamma=1 . e-12$} & \multicolumn{3}{|c|}{ ITER=121 } & \multicolumn{3}{c|}{$\gamma=1 . e-4$} & ITER=66 \\
\hline
\end{tabular}
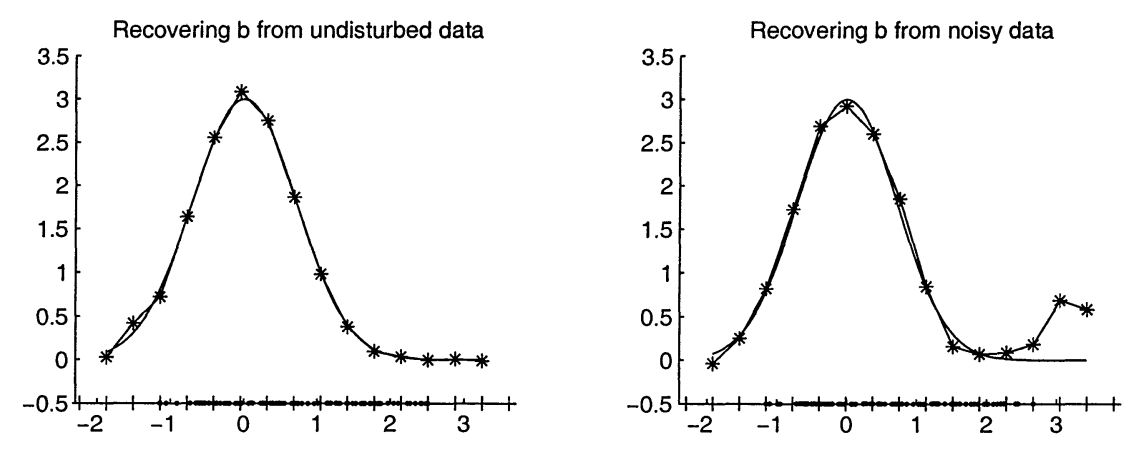

Figure 1. Example 1. $b(y)=3 \exp \left(-y^{2}\right)$.

From our point of view, in this case, the numerical results are qualitatively good in the interval $I$ defined by the data $\eta_{i j}$, even under the presence of a relatively high level of noise (up to 10\%). As one can expect, in the safety zone $I_{w} \backslash I$, the approximation becomes worse, but it seems still acceptable.

Example 2 We consider the recuperation of $a(x) b(y)$ with $a(x)=$ $\sin (\pi x / 4)$ and $b(y)=3 \exp \left(-y^{2}\right)$ from the data corresponding to the 
source terms $u_{j}$ given by $(12)$ with $\left(i_{j}, k_{j}\right)$ taking the values $(1,1),(1,8)$, $(2,2),(3,5),(5,6)$ and $(15,4)$. No explicit noise is included here (i.e. $\delta=0$ ). In Figure 2, we show the computed results for $\epsilon=1 . e-12$ and $\varepsilon=2 . e-9$, assuming $\hat{a}_{1} \geq 1 . e-12$. This figure contains four graphics. On the top, the numerical approximation (obtained after 151 iterations) and the exact nonlinearity $a(x) b(y)$ appear on the left and on the right, respectively. On the bottom, the relative errors

$$
\frac{\left|\check{a}\left(x_{i}\right) \check{b}\left(y_{j}\right)-a\left(x_{i}\right) b\left(y_{j}\right)\right|}{\max \left(1,\left|a\left(x_{i}\right) b\left(y_{j}\right)\right|\right)}
$$

are shown.
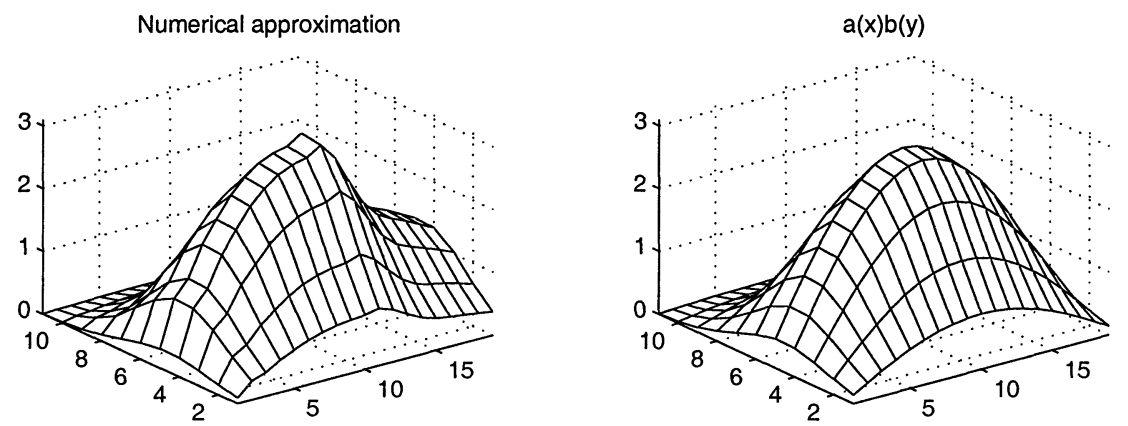

Relative error for $\mathrm{x}$ in $(0,4)$

Relative error for $\mathrm{x}$ in $(0,3.4]$
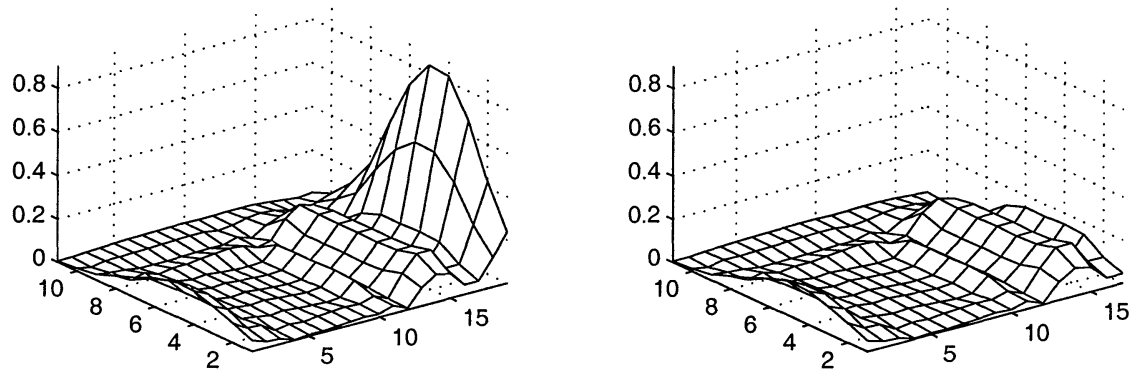

Figure 2. Example 2. $a(x)=\sin (\pi x / 4)$ and $b(y)=3 \exp \left(-y^{2}\right)$.

In this example, we think that the computed results are relatively satisfactory when $x \in(0,3.4]$ and they can be improved in $(3.4,4)$. In our opinion, the deterioration in this zone can be due to the lack of information there: let us remind that $\omega_{\text {in }}$ and $\omega_{\text {out }}$ are both included in $[0,2.1)$, while $\Omega=(0,4)$. This difficulty does not appear when the nonlinear term is independent of the space variable $x$. 


\section{References}

[1] Fabre, C., Puel, J. P., and Zuazua, E. (1995). Approximate controllability of the semilinear heat equation. Proc. Royal Soc. Edinburgh, 125A:31-61.

[2] Fernández, L. A. and Pola, C. (2001). Identification of a quasilinear parabolic equation from final data. Int. J. Appl. Math. Comput. Sci., 11(4):859-879.

[3] Fernández, L. A. and Zuazua, E. (1999). Approximate controllability for the semilinear heat equation involving gradient terms. J. Optimization Theory 6 Appl., 101(2):307-328.

[4] Isakov, V. (1998). Inverse Problems for Partial Differential Equations. Springer, New York-Berlin-Heidelberg.

[5] Ladyzhenskaya, O. A., Solonnikov, V. A., and Ural'tseva, N. N. (1968). Linear and Quasilinear Equations of Parabolic Type. Am. Math. Soc, Providence, R. I. 